\title{
Trend analysis of lab tests requisitions of COVID-19 prognostic biomarkers at a clinical chemistry reference laboratory-an observational study
}

Sibtain Ahmed

Aga Khan University, sibtain.ahmed@aku.edu

Farooq Ghani

Aga Khan University, farooq.ghani@aku.edu

Follow this and additional works at: https://ecommons.aku.edu/pakistan_fhs_mc_pathol_microbiol

Part of the Pathology Commons

\section{Recommended Citation}

Ahmed, S., Ghani, F. (2020). Trend analysis of lab tests requisitions of COVID-19 prognostic biomarkers at a clinical chemistry reference laboratory-an observational study. Annals of Medicine and Surgery, 60, 522-525.

Available at: https://ecommons.aku.edu/pakistan_fhs_mc_pathol_microbiol/1298 
Short Communication

\title{
Trend analysis of lab tests requisitions of COVID-19 prognostic biomarkers at a clinical chemistry reference laboratory-an observational study
}

\author{
Sibtain Ahmed, Farooq Ghani \\ Pathology and Laboratory Medicine, Aga Khan University, Stadium Road, Karachi, 74800, Pakistan
}

\section{A R T I C L E I N F O}

\section{Keywords:}

COVID-19

C-reactive protein

Procalcitonin

Lactate dehydrogenase

Ferritin

Prognosis

\begin{abstract}
A B S T R A C T
Background: Biochemical parameters, such as C-reactive protein (C-RP), Procalcitonin (PCT), Lactate dehydrogenase (LDH) and Ferritin are associated with COVID-19 severity and prognosis. This trend analysis of COVID-19 prognostic biomarker requisitions is aimed at providing a reflection of the clinical practices adopted amidst the peak of the pandemic. This knowledge of the prognostic biomarkers utilized, will further aid to classify patients based on their risk, for optimal resource allocation and improve outcomes.

Material \& methods: This cross-sectional study was conducted at the clinical chemistry laboratory at the Aga Khan University Hospital (AKUH), Karachi Pakistan. A team consisting of two clinical chemistry consultants reviewed the COVID-19 prognostic biomarkers tests performance data from March to July 2020 using the integrated laboratory information management system (ILMS). The test statistics of March to July 2020 when COVID-19 cases were on the rise in the country were compared with those of the same months from 2019. The changes in the test groups were expressed in percentages. Microsoft Excel for windows 2019 was used for data analysis. Results: The total specimens received for testing in $2020(\mathrm{n}=574,092)$ showed a percent decline of approximately ( - ) 33\% compared to $2019(\mathrm{n}=858,756)$. Contrary to the overall decline in volumes an upward surge was noted for the prognostic biomarkers. From March to July, the highest percent change was noted for LDH $(+155.1 \%)$, followed by PCT $(+66.7 .1 \%)$, C-RP $(+26.5 \%)$ and Ferritin $(+22.1 \%)$ in 2020 compared to the same time frame in 2019. The highest percent change was noted in June.

Conclusion: During the COVID 19 pandemic, a significant increase in utilization of laboratory services was seen for COVID-19 prognostic markers.
\end{abstract}

\section{Introduction}

The Coronavirus Disease 2019 (COVID-19) pandemic, a respiratory and systemic viral disease, caused by severe acute respiratory syndrome coronavirus 2 (SARS-CoV-2), emerged on the global health scenario in late December 2019 and becoming a global pandemic in 2020 [1]. Clinical laboaratories have played a critical role in managing the pandemic [2]. Even though the clinical laboratories continued their operations 24/7, the lockdown and restrictions imposed by most countries to decrease the risk of contagion, have affected the course of routine medical services [3].

In this era of unprecedented health crisis, in addition to rapid and accurate diagnostic tests, the clinical laboratory specialists were in search for reliable biomarkers associated with COVID-19 disease progression, in order to stratify high risk subjects, particularly in resource constrained setups for optimal intensive care [4].
COVID-19 patients are classified into mild, moderate, severe, and critical disease [5]. Various studies have reported biochemical parameters, such as C-reactive protein (C-RP), Procalcitonin (PCT), Lactate dehydrogenase (LDH) and Ferritin to be associated with COVID-19 severity and prognosis [3]. The increase in inflammation markers and acute phase reactants are linked with the underlying systemic vasculitic processes and the cytokine storm that cause most parenchymal lesions in vital organs [3].

On one end, amid the COVID-19 pandemic, the delay of non-urgent health services and subsequent decline in the number of patients due to concern of contagion, is expected to lead to a significant drop in the overall routine biochemical test requisitions, while on the other, a rapid surge in the test requests for the COVID-19 prognostic biomarkers is projected. At our institute, a tertiary care hospital in the metropolis of Karachi, during the lockdown and restrictions, all elective, semi-elective procedures and non-essential procedures were cancelled. From April to

\footnotetext{
* Corresponding author.

E-mail addresses: sibtain.ahmed@aku.edu (S. Ahmed), farooq.ghani@aku.edu, farooq.ghani@aku.edu (F. Ghani).
} 
June 2020, our pediatric surgery department, performed only one-third the volume compared to the preceding three months [6]. Moreover, the number of clinics in each specialty was cut down and tele-clinics were also introduced from beginning of April [7].

The aim of this study was to investigate the trend in COVID-19 prognostic biomarkers requisitions including C-RP, PCT, LDH and Ferritin, at a high-volume clinical chemistry laboratory in Pakistan during the peak pandemic months. Furthermore, the test statistics of March to July 2020 when COVID-19 cases were on the rise were compared with those of 2019. While much was unknown about the course of COVID-19 disease, the clinicians utilized some of the lessons learnt from previous experiences and knowledge for prognostic biomarker selection. This trend analysis of COVID-19 prognostic biomarker requisitions is aimed at providing a reflection of the clinical practices adopted amidst the peak of the pandemic. This knowledge of the prognostic biomarkers utilized, will further aid to classify patients based on their risk, for optimal resource allocation and improve outcomes.

\section{Methods}

This retrospective observational study was conducted at the clinical chemistry laboratory at the Aga Khan University Hospital (AKUH), Karachi Pakistan, which serves as a national reference lab for the country. With its growing network of more than 260 phlebotomy centers and outreach laboratories spread across Pakistan, it caters to test requests from all the provinces. The laboratory operates to highest standards of quality and was the first lab to be accredited by College of American Pathologists (CAP) as well as Joint Commission International Accreditation (JCIA).

A team consisting of two clinical chemistry consultants reviewed the COVID-19 prognostic biomarkers tests performance data from March to July 2020 using the integrated laboratory information management system (ILMS). The test statistics of March to July 2020 when COVID-19 cases were on the rise in the country were compared with those of the same months from 2019.

Frequencies and percentages were analyzed for confirmed cases of covid-19, active cases of covid-19, deaths, recoveries, total specimen count and total prognostic biomarkers specimen count of 2019 and 2020 respectively. The test requisition statistics of prognostic biomarkers volumes for March to July in 2019 and 2020 were compared; and the changes in the test groups were expressed in terms of percent change alongside its graphical representation. Microsoft Excel for windows 2019 was used for data analysis.

\section{Results}

A descriptive detail of the total confirmed cases, active cases, deaths and recoveries according to the data collected by the Government of Pakistan alongside the change in total specimens received and the four prognostic biomarkers specimens received based on a comparison between 2019 and 2020 for the months from March to July is shown in Table 1 [8].

Furthermore, the total specimens received for testing in 2020 ( $\mathrm{n}=$
$574,092)$ showed a percent decline of approximately (-) 33\% compared to 2019 ( $n=858,756$ ). Contrary to the overall decline in volumes an upward surge was noted for the prognostic biomarkers i.e. the total specimen volume received for testing in $2020(n=71,548)$ showed $41.4 \%$ increase compared to $2019(n=50,601)$. In totality, from March to July, the highest percent change was noted for LDH (+155.1\%), followed by PCT $(+66.7 .1 \%)$, C-RP $(+26.5 \%)$ and Ferritin $(+22.1 \%)$ in 2020 compared to the same time frame in 2019. The highest percent change was noted in June for all the biomarkers, the highest being LDH $(+483.1)$ followed by Ferritin, PCT and C-RP respectively.

On further stratification into monthly volumes, the percent change noted for these biomarkers in 2020 compared to 2019 is elaborated in Table 2. Fig. 1 presents a graphical depiction of trend analysis of the four prognostic biomarkers test volumes recorded in 2020 compared to the subsequent months in 2019.

\section{Discussion}

The test requisition data shows a general surge in the four prognostic biomarkers utilization amid the COVID-19 pandemic. This study depicts that PCT demonstrated a positive trend right from March 2020 compared to the other three biomarkers, which were initially lower compared to 2019 in March and April. PCT is an established biomarker to differentiate bacterial from viral infections, and a meta-analysis reported a $\sim 5$-fold higher risk of severe COVID-19 infection associated with elevated PCT $[9,10]$. However, a paradigm shift was observed in May 2020 when a steady increase in test requests was noted for all the four biomarkers.

This variation in trend could be linked to several reasons, for instance May 2020 was regarded as the month when the cases of COVID-19 exhibited a rapid upheave in Pakistan [11]. Additionally, the strict lockdown imposed in late March restricted patient movements leading to a decline in test volumes, which gradually improved as the restrictions were subsequently relaxed in the following months beginning around May 10, 2020 [12]. Additionally, the hospital to which the

Table 2

Percent change in COVID-19 prognostic biomarkers volumes for March to July in 2019 and 2020.

\begin{tabular}{lllll}
\hline & C-RP & LDH & PCT & Ferritin \\
\hline March 2019 (n) & 5935 & 974 & 1199 & 2920 \\
March 2020 (n) & 4069 & 687 & 1292 & 1552 \\
Change (\%) & $-\mathbf{3 1 . 4}$ & $-\mathbf{2 9 . 5}$ & $+\mathbf{7 . 8}$ & $-\mathbf{4 6 . 8}$ \\
April 2019 (n) & 5680 & 948 & 1011 & 3174 \\
April 2020 (n) & 3648 & 1115 & 1226 & 1690 \\
Change (\%) & $-\mathbf{3 5 . 8}$ & $+\mathbf{1 7 . 6}$ & $+\mathbf{2 1 . 3}$ & $-\mathbf{4 6 . 8}$ \\
May 2019 (n) & 4944 & 894 & 1026 & 2758 \\
May 2020 (n) & 5238 & 1743 & 1603 & 2782 \\
Change (\%) & $+\mathbf{5 . 9}$ & $+\mathbf{9 4 . 9}$ & $+\mathbf{5 6 . 2}$ & $+\mathbf{0 . 8}$ \\
June 2019 (n) & 4439 & 872 & 1041 & 2435 \\
June 2020 (n) & 11,395 & 5085 & 2686 & 6436 \\
Change (\%) & $+\mathbf{1 5 6 . 7}$ & $+\mathbf{4 8 3 . 1}$ & $+\mathbf{1 5 8 . 0}$ & $+\mathbf{1 6 4 . 3}$ \\
July 2019 (n) & 5110 & 967 & 1188 & 3086 \\
July 2020 (n) & $\mathbf{8 6 6 4}$ & 3247 & 2305 & 5085 \\
Change (\%) & $+\mathbf{6 9 . 5}$ & $+\mathbf{2 3 5 . 8}$ & $+\mathbf{9 4 . 0}$ & $+\mathbf{6 4 . 8}$ \\
\hline
\end{tabular}

Table 1

COVID-19 cases in Pakistan and the trend of change in total number of specimens and prognostic biomarkers specimens' data of March to July 2020 compared to same time period in 2019.

\begin{tabular}{|c|c|c|c|c|c|c|c|c|}
\hline Months & $\begin{array}{l}\text { Confirmed Cases of } \\
\text { COVID-19 (n) }\end{array}$ & $\begin{array}{l}\text { Active Cases of } \\
\text { COVID-19 (n) }\end{array}$ & $\begin{array}{l}\text { Deaths } \\
\text { (n) }\end{array}$ & $\begin{array}{l}\text { Recoveries } \\
\text { (n) }\end{array}$ & $\begin{array}{l}\text { Total Specimen } \\
\text { count } 2019(\mathrm{n})\end{array}$ & $\begin{array}{l}\text { Total Specimen } \\
\text { count } 2020(\mathrm{n})\end{array}$ & $\begin{array}{l}\text { Total Prognostic } \\
\text { Biomarkers Specimen } \\
2019(n)\end{array}$ & $\begin{array}{l}\text { Total Prognostic } \\
\text { Biomarkers Specimen } \\
2020 \text { (n) }\end{array}$ \\
\hline March & 2039 & 1931 & 26 & 82 & 188,710 & 125,321 & 11,028 & 7600 \\
\hline April & 16,817 & 12,117 & 385 & 4315 & 192,966 & 81,574 & 10,813 & 7679 \\
\hline May & 72,460 & 44,834 & 1543 & 26,083 & 153,898 & 95,857 & 9622 & 11,366 \\
\hline June & 213,470 & 108,273 & 4395 & 100,802 & 146,784 & 132,017 & 8787 & 25,602 \\
\hline July & 279,146 & 25,149 & 5970 & 248,027 & 176,398 & 136,323 & 10,351 & 19,301 \\
\hline
\end{tabular}




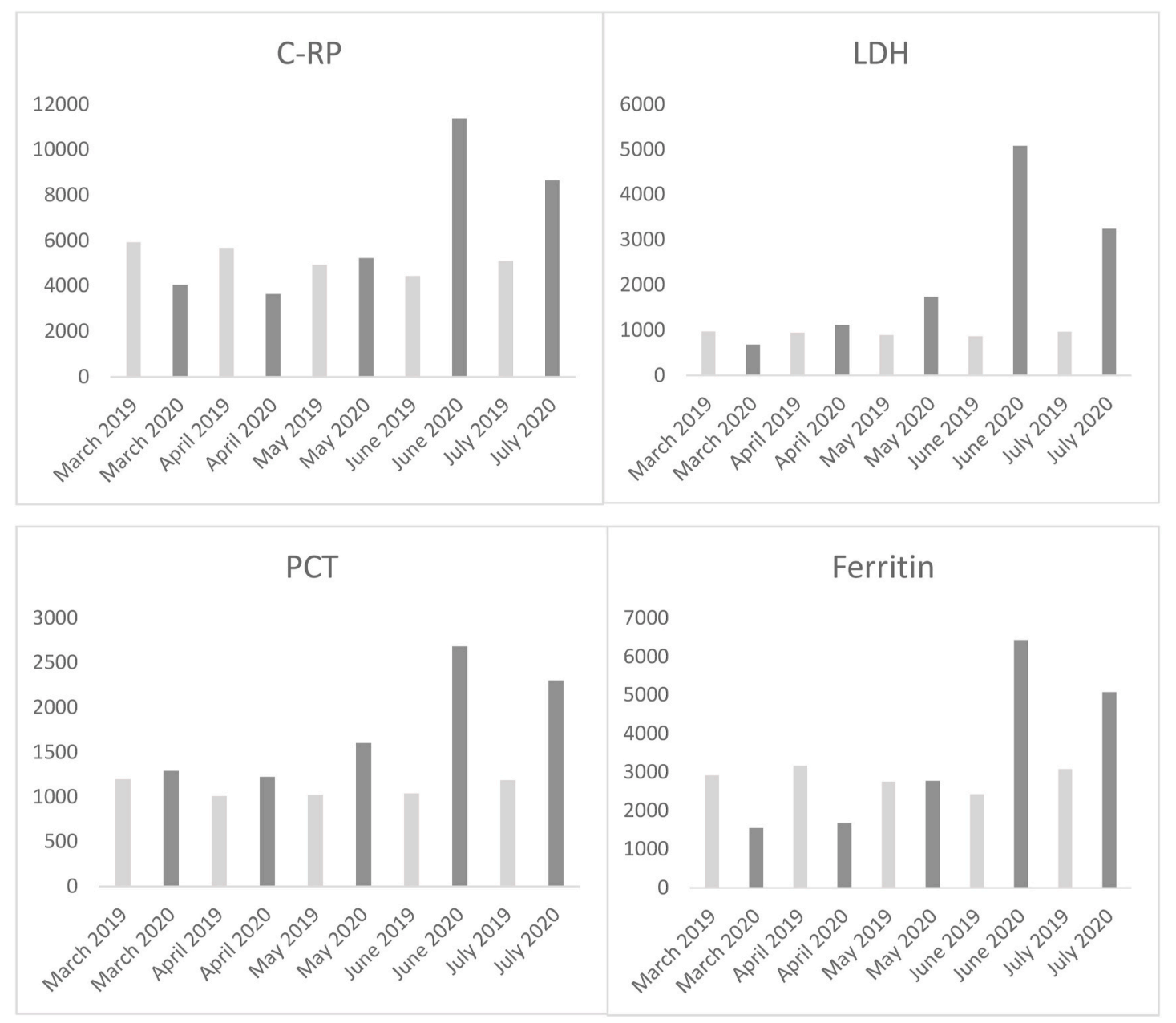

Fig. 1. A comparison of COVID-19 prognostic biomarkers volumes for March to July in 2019 and 2020.

laboratory is affiliated with, started a dedicated COVID-19 inpatient facility which further enhanced test requisition flow. This establishment alongside the tele-clinics improved routine patient inflow and the surge in test requests was evident.

The highest number of active cases in Pakistan were recorded in June, which is in agreement with our data as an average of $+240.5 \%$ hike in prognostic biomarkers test utilization was noted, which was significantly higher than the previous three months of 2020. Compared to the other three prognostic markers, significantly higher utility of LDH was noted. LDH is a cytoplasmic glycolytic enzyme found in almost every tissue, and its elevation generally indicates tissue damage. The role of elevated LDH has been previously well established in patients infected with MERS-CoV, H7N9, and H5N1 [13]. It has been widely accepted as an independent predictor of mortality for patients with severe acute respiratory syndrome and H1N1 infection [14]. Its previous strong association with prognosis in acute respiratory distress syndromes could be one reason for the increased requisition by physicians.

On further evaluation, the number of requisitions declined significantly during July compared to June 2020 . This trend is in line with the COVID-19 country statistics, as Pakistan demonstrated a flattening of the disease curve in July 2020 [15].

The total specimen count in 2020 was lower during the peak pandemic months, a major contributor being lock down and movement restrictions. Even though, the hospital conceived a recovery plan for medical and surgical services and switched from 'crisis mode' to 'back to usual' the overall volumes remained low compared to the time span of 2019 for the duration of this study. The potential reasons being the apprehensions of the patients and their families alongside the fear of contracting the virus from visiting medical and diagnostic facilities, while waiting in patient areas or being exposed while being treated, that have not been adequately alleviated despite the plateau in the number of cases. Subsequently, a significant drop in the overall routine biochemical test requisitions, while on the other, a rapid surge in the test requests for the COVID-19 prognostic biomarkers was evident as shown by this study.

Several guidelines were issued in response to pandemic preparedness that primarily focused on containment, treatment and diagnostic modalities. Meanwhile, there was also a dire need for early risk stratification systems and biomarkers to predict disease progression and to identify high-risk patients at an early stage of the infection. During the COVID 19 pandemic while there was a significant reduction in overall laboratory requisitions owing to reduction in out-patient clinics and elective procedures, a significant increase in utilization of laboratory services was seen for C-RP, PCT, LDH and Ferritin. This elevated trend of prognostic biomarkers requisitions showed that while much was unknown about the course of COVID-19 disease, the clinicians utilized some of the lessons learnt from previous experiences and knowledge of MERS-CoV, H7N9, and H5N1 and as more details regarding COVID clinical course, pathologies and possible useful interventions emerged in literature, clinicians utilized the laboratory services to good effect. This approach can optimize management goals, and overcome the shortage of medical and material resources which was particularly evident amidst this global emergency. As identification of reliable outcome predictors in COVID-19 is of paramount importance for improving patient's management, while this specific objective was beyond the scope of this study, various studies have shown improved outcome, based on the 
prognostic utility of abnormal inflammatory and organ injury circulating biomarkers $[16,17]$.

There were certain limitations of this study, as the evaluation of patients' information/database was not performed, this study cannot demarcate between tests requisitions specifically pertaining to COVID19 proven cases and those requested in routine clinical care of other disorders.

\section{Conclusion}

As the data on the use of selective biomarkers becomes available which showed more affective patient management and improved outcomes, it has now become a standard practice to test for these prognostic biomarkers once a patient tests positive for COVID-19. Clinical laboratories should be prepared for the optimal inventory management in the pandemic era owing to the varying trend of biomarkers utilizations, as well as potential interruption of international trade. Moreover, delayed health care requirements are further expected to increase the work flow to the hospitals acutely as the restrictions are lifted. Therefore, the national case statistics should be carefully followed and resources of both personnel and materials should be pre-planned by the laboratory stakeholders.

\section{Funding}

The authors did not receive any funding for this project

\section{Ethical approval}

Not applicable as the research work is based on trend analysis of laboratory volumes and does not involve intervention/interaction with human subjects or related information.

\section{Consent}

Not applicable.

\section{Author contribution}

SA performed the data analysis and write-up of the work. FG conceived the idea, collected the data and coordinated the writing of the paper and reviewed the final draft. All the authors have accepted responsibility for the entire content of this submitted manuscript and approved submission.

\section{Registration of research studies}

Not applicable.

\section{Guarantor}

The Guarantor is the one or more people who accept full responsibility for the work and/or the conduct of the study, had access to the data, and controlled the decision to publish.

\section{Declaration of competing interest}

None.

\section{References}

[1] L. Jafri, S. Ahmed, I. Siddiqui, Impact of COVID-19 on laboratory professionals-A descriptive cross sectional survey at a clinical chemistry laboratory in a developing country, Annals of Medicine and Surgery (2020 Jul 18), https://doi.org/10.1016/j. amsu.2020.07.022.

[2] G. Lippi, M. Plebani, The critical role of laboratory medicine during coronavirus disease 2019 (COVID-19) and other viral outbreaks, Clin. Chem. Lab. Med. (2020 Mar 19) 1, https://doi.org/10.1515/cclm-2020-0240.

[3] S. Ahmed, L. Jafri, H. Majid, A.H. Khan, F. Ghani, I. Siddiqui, Challenges amid COVID-19 times-Review of the changing practices in a clinical chemistry laboratory from a developing country, Annals of Medicine and Surgery (2020 Jun 6), https://doi.org/10.1016/j.amsu.2020.06.004.

[4] G. Ponti, M. Maccaferri, C. Ruini, A. Tomasi, T. Ozben, Biomarkers associated with COVID-19 disease progression, Crit. Rev. Clin. Lab Sci. (2020 Jun 3), https://doi. org/10.1080/10408363.2020.1770685, 1-1.

[5] Diagnosis and treatment protocol for novel coronavirus pneumonia (trial version 7) released by National Health Commission \& National Administration of Traditional Chinese Medicine, Chin Med J (Engl) 133 (2020) 1087-1095, https://doi.org/ 10.1097/CM9.0000000000000819.

[6] S.H. Qazi, S.A. Dogar, L.R. Hamid, A.N. Pirzada, A. Saleem, J.K. Das, Continuing and ensuring surgical care for children during COVID and post-COVID crisis, J. Pediatr. Surg. (2020). https://dx.doi.org/10.1016/j.jpedsurg.2020.08.012.

[7] M.H. Roshan-e-Shahid Rana, S.A. Ather, Change in surgical practice amidst COVID 19; example from a tertiary care centre in Pakistan, Annals of Medicine and Surgery 54 (2020 Jun) 79.

[8] COVID-19 cases in Pakistan. http://covid.gov.pk/stats/pakistan. Accessed on 8th August 2020.

[9] S. Ahmed, I. Siddiqui, L. Jafri, M. Hashmi, A.H. Khan, F. Ghani, Prospective evaluation of serum procalcitonin in critically ill patients with suspected sepsisexperience from a tertiary care hospital in Pakistan, Annals of medicine and surgery 35 (2018 Nov 1) 180-184, https://doi.org/10.1016/j.amsu.2018.10.004.

[10] G. Lippi, M. Plebani, Procalcitonin in patients with severe coronavirus disease 2019 (COVID-19): a meta-analysis, Clin. Chim. Acta 505 (2020) 190-191, https://doi. org/10.1016/j.cca.2020.03.004.

[11] R. Ahmed, S. Ahmed, Real-time forecast of final outbreak size of novel coronavirus (COVID-19) in Pakistan: a data-driven analysis, Available at: SSRN 3594111, https://dx.doi.org/10.2139/ssrn.3594111, 2020 May 6.

[12] S. Chandir, D.A. Siddiqi, H. Setayesh, A.J. Khan, Impact of COVID-19 lockdown on routine immunisation in Karachi, Pakistan, The Lancet Global Health 8 (2020 Jun 29) 1118-1120, https://doi.org/10.1016/S2214-109X(20)30290-4.

[13] J. Shi, Y. Li, X. Zhou, Q. Zhang, X. Ye, Z. Wu, X. Jiang, H. Yu, L. Shao, J.W. Ai, $\mathrm{H}$. Zhang, Lactate dehydrogenase and susceptibility to deterioration of mild COVID-19 patients: a multicenter nested case-control study, BMC Med. 18 (1) (2020 Dec) 1-6, https://doi.org/10.1186/s12916-020-01633-7.

[14] X. Xi, Y. Xu, L. Jiang, A. Li, J. Duan, B. Du, C.C.C.T. Chinese, Hospitalized adult patients with 2009 influenza A (H1N1) in Beijing, China: risk factors for hospital mortality, BMC Infect. Dis. 10 (1) (2010) 256, https://doi.org/10.1186/\%2F14712334-10-256.

[15] Pakistan's stunning flattening of Covid-19 curve. https://tribune.com.pk/st ory/2256760/pakistans-stunning-flattening-of-covid-19-curve. (Accessed August 2020). Accessed on 5th.

[16] S. Figliozzi, P.G. Masci, N. Ahmadi, L. Tondi, E. Koutli, A. Aimo, K. Stamatelopoulos, M.A. Dimopoulos, A.L. Caforio, G. Georgiopoulos, Predictors of adverse prognosis in COVID-19: a systematic review and meta-analysis, Eur. J. Clin. Invest. (2020 Oct 1), e13362.

[17] L. Yan, H.T. Zhang, J. Goncalves, Y. Xiao, M. Wang, Y. Guo, C. Sun, X. Tang, L. Jing, M. Zhang, X. Huang, An interpretable mortality prediction model for COVID-19 patients, Nature Machine Intelligence (2020 May 14) 1-6. 